\title{
Prevalence of Gonorrhea among Pregnant Women in Nigeria: Reproductive Health Implication
}

\author{
Goodluck Azuonwu ${ }^{\mathrm{a}}$, Awonemika Caroline Arimie ${ }^{\mathrm{b}}$ \\ ${ }^{\text {a }}$ Email: goodator2002@yahoo.com \\ ${ }^{a}$ Department of Nursing Science, University of Port Harcourt, Rivers State, Nigeria \\ ${ }^{a}$ Department of Human Kinetics, Health and Safety Studies, Ignatius Ajuru University of Education, Rivers State Nigeria
}

\begin{abstract}
Sexually transmitted diseases (STDs) including gonorrhea are a major reproductive health issue. Gonorrhea is a disease caused by Neisseria gonorrhea bacteria and is the one of the causes of PID in women. The global prevalence of gonorrhea is estimated to be at $0.9 \%$ which is high. This paper sought to provide updated information on the prevalence of gonorrhea infection and to discuss the reproductive health implications. Methods: literature search using PubMED, Google Scholar and Medline was conducted. Studies included were cross sectional studies reporting the prevalence of gonorrhea in Nigeria, conducted from 2000 to 2021 . The population from the various studies analyzed was 1725 women of reproductive age between 15 and 45 years. Findings: the prevalence of gonorrhea was found to be $2.0 \%$ in the East, $5.2 \%, 5.0 \%$ and $0.7 \%$ in the South, $3.4 \%$ in the West and $16.3 \%$ in the North. The reproductive health implication is that untreated STIs leads to pelvic inflammatory disease (PID) and may further result to infertility and part of the goal for most couple is to reproduce. Treating PID is one of the most difficult task for clinicians thus except gonorrhea is combated the problem of infertility will not be ameliorated and thereby achieving safer birth and better reproductive health outcomes will almost be near impossible.
\end{abstract}

Key Words: Sexually Transmitted Disease; Gonorrhea; Reproductive Health

\section{Introduction}

Sexually transmitted diseases (STDs) including gonorrhea are a major reproductive health issue. Gonorrhea is disease caused by bacteria and transmitted through sexual and non- sexual contacts. Gonorrhea is sexually transmitted by Neisseria gonorrhea (Hethcote \&Yorke 2014). The global prevalence of these Gonorrhea is significantly high (Rowley (2019) estimated that the global prevalence of gonorrhea is $0.9 \%$ and this infection is the leading cause of acute urogenital deformities, sepsis, pelvic inflammatory disease, infertility, adverse birth outcomes and other serious complication if left untreated. (Okonko et al. 2012; Omatola et al. 2020). Despite the various interventions designed and implemented to stop the spread of gonorrhea, it has remained a challenging issue. According to the World Health Organization (WHO,2012), in Sub Saharan Africa about 21.1 million new cases of gonorrhea occur yearly most cases occurring among women of reproductive age between the age of 15 to 45 years (Hook, 2017; Culbreth et al. 2020).

Gonorrhea can be contracted when an individual have unprotected sex with an infected person. The gonorrhea bacteria infect all external and internal exposed genital area either in the victim that come into contact with infected semen or vaginal fluid during sex (Burnett et al., 2012; Mitchell et al., 2021). It can survive in the rectum, cervix, urethra, 
throat and eyes for a long time (Kreisel \& Spicknall et al. 2018). This infection spreads from the vagina to the anus without the woman even having sex and as a result this spread could lead to mother to child transmission in pregnant women (Center for Disease Control (CDC, 2021). According to Achalu (2004), some infected people can have signs and symptoms within two weeks after infection while others may not present with any symptom (asymptomatic). Available evidence suggest that the signs and symptoms of gonorrhea differ both in males and females (Workowski, 2013; Danby et al., 2016; Cornelisse et al., 2017). In men these symptoms include: unusual white, yellow or green discharge from the tip of the penis, pain or a burning sensation when urinating; inflammation of the foreskin and pain or tenderness in the testicles or prostate gland (Edem, 2013; Keshinro et al., 2015). In contrast infected women release green or yellowish thick usual discharge from the vagina, they experience pain when urinating or pain and tenderness in the lower abdomen and bleeding between periods or heavier periods but this is less common (Cohen \& Cannon, 1999). In newborns gonorrhea affect the eyes during the first two weeks. The eyes become red and swollen, and have thick pus-like discharge conjunctivitis.

However, the situation is more complicated and serious in women, with 50-80\% of lower genital tract $\mathrm{N}$. gonorrhoeae infections (gonococcal cervicitis) remaining asymptomatic (Craig et al., 2015). The movement of this bacteria from the cervix to the fallopian tubes occurs in up to $45 \%$ of gonococci-infected women leading to pelvic inflammatory disease (PID: inflammation of the uterus, fallopian tubes or ovaries (Atalabi et al., 2013; Vincent \& Jerse , 2019) Other sequelae include adverse pregnancy outcomes (pre-term birth, spontaneous abortion, stillbirth, low infant birth weight, ectopic pregnancy, postpartum endometriosis or sepsis, ophthalmianeonatorum), infertility, and disseminated gonococcal infection (Reekie et al., 2019;Marrazzo, \& Dionne-Odom, 2021). Infection with N. gonorrhoeae also increases HIV replication, transmission, and infection (Adachi et al., 2015; Barbee et al., 2017).

The populations at risk of contracting gonorrhea are mostly individuals that are sexually active, women with multiple sex partners, pregnant women, people living with Human Immunodeficiency Virus (HIV), men who have sex with men and children as a result of mother to child transmission (Ramadhandi et al., 2017). Globally, about 78 million people are infected with gonorrhea (WHO, 2016; CDC, 2016). Despite the efforts made to reduce this infection; gonorrhea has continued to exert a high burden on reproductive age women in resource constrained regions including Nigeria. This infection is transmitted from one person to another through vaginal, oral, or anal sex. Men have a $20 \%$ risk of getting the infections from a single act vaginal intercourse with an infected woman; the risk for men that have sex with men is higher. Women have a $60-80 \%$ risk of getting the infection from a single act of vaginal intercourse with an infected man.

Nigeria is among the most populous nations in the world and more than half of its population is sexually active between 15- 45 years. A rapid increase in the number of people living with gonorrhea could result to an epidemic with severe health, social and economic consequences if this infection is not prevented (Chiwa et al., 2019). For example the cost of treating gonorrhea in developing countries is approximately 21.66 USD per person, estimated cost of administering was estimated to be in excess of 187 USD and testing alone cost about \$1.4 billion USD (Korenromp, 2017). While it is difficult to determine costs directly attributable to antimicrobial resistance (AMR), however Tapsel 
(2005) conducted an analysis to estimate the economic impact of AMR the report revealed that AMR contributed to over 3 million treatment failures worldwide each year and cost over 500 million USD per year. Additionally most recent estimates predicts an increased number of these infections with associated HIV cases due to emerging AMR, thus the treatment of this infection will cost above 378.2 million USD in the next ten years (Chesson et al., 2017).

The goal of the global health sector strategy is to reduce the incidence of STIs in the coming decade (WHO, 2016). Particularly in countries where diagnostic testing and screening for gonorrhea are readily available, prompt detection and effective treatment prevent sequelae and further transmission. However, the challenge of providing effective treatment is under threat, as $\mathrm{N}$. gonorrhea has successively acquired antimicrobial resistance to each antimicrobial agent used and recommended for treatment (Unemo et al., 2026). Influence of emerging resistance to cephalosporins and macrolides and a dwindling antimicrobial development threatens to undermine gonorrhea control and worsen ongoing management challenges.

In order to support the implementation of prevention and control programs and monitor the progress made toward reducing the incidence of these infection in Nigeria, it is important to understand the prevalence of these infections in Nigeria and the reproductive health implication as it would help to guide clinical decision making, public resource allocation, and optimization of intervention protocols. Although several scholars (Mbamara et al., 2011; Bukar et al., 2009; Ugochukwu et al., 2015; Opene et al., 2019) have investigated the prevalence of gonorrhea in different states in Nigeria, overall data summarizing the National prevalence of gonorrhea is scare or non- existent.

\subsection{Aim of the Review}

This review sought to provide updated information on the prevalence of gonorrhea infection and to discuss the reproductive health implications.

1.2 Objectives of the Review

- Review literatures on the prevalence of gonorrhea

- Discuss the reproductive health implication

- $\quad$ Suggest a way forward

\section{Review of Related Literature}

\subsection{Prevalence of Gonorrhea}

Gonorrhea prevalence from the four regions (East, West North and South) in Nigeria: In a cross sectional study conducted by Nsofor and Eletuah (2017) from the East gonorrhea prevalence was found to be $2.0 \%$ (low)while in the South, Usanga et al., (2010), Wariso and Oboro (2013) and Edem et al., (2013) reported 5.2\%, 5.0\% and 0.7\% prevalence respectively. The West had a low prevalence of 3.4\% Ugochukwu et al., (2015) as compared to the North which had a very high prevalence of $16.3 \%$ as revealed in a study conducted by Jatau et al., (2003). Although the study 
from the north was the most recent study the reviewer found. However, the reviewer acknowledges that this may not reflect the current prevalence of gonorrhea from the northern region.

\subsection{Implication for Reproductive Health}

Gonorrhea is an STI with severe reproductive health consequences that are easily preventable and treatable; however, they continue to pose as serious threats. Prevention and control of gonorrhea is vital for all women given the negative reproductive health outcomes associated with the infection. Moreover, proper diagnosis and screening including periodic follow-up screening plays a significant role in controlling the spread. Although treatment and prevention messages have been on for some decades, which could be a reason for the overall decline observed in this paper, however, the high prevalence rate observed in some regions suggest that several women in these regions where the prevalence is high may not be taking advantage of the public health messages to avoid risky behaviors that predisposes them to gonorrhea.

The highest prevalence of gonorrhea in the review was found in the North. The differences in the regional prevalence mainly reflect the risk profiles of the populations included in the studies. The high prevalence of gonorrhea in the north is a particular concern as this implies that more cases of fetal/neonatal mortality and morbidity will be seen because of mother to child transmission.

Despite the decreasing trend of gonorrhea observed especially in the overall population in terms of the years, the high prevalence seen in the northern region was three times higher than that of the south, the regional differences observed in women could be because of differences in the strength of programs for primary prevention, quality of followup programs and availability of interventions in the health system. Effective prevention targeted towards women at risk should involve multiple interventions to reduce risky behavior, reduce opportunities for transmission, and lower biological susceptibility of transmitting or acquiring infection. Despite the availability of evidence-based behavioral and biomedical tools that reduce gonorrhea transmission or risk, the challenge for federal agencies and state and local partners responsible for delivering prevention services, is understanding which combinations of prevention interventions will produce the most robust results in specific high-risk communities.

Furthermore, paper highlights the national prevalence of the disease in regions across Nigeria. Given that complications of gonorrhea infection disproportionately affect women of all ages and have severe implication for women of reproductive age owing to the substantial costs associated with the treatment, it is important that clinicians conduct adequate screening recommendations for women to help reduce the emergence of new cases and the spread. Untreated STIs leads to pelvic inflammatory disease (PID) and may further result to infertility and part of the goal for most couple is to reproduce. Treating PID is one of the most difficult task for clinicians thus except gonorrhea is combated the problem of infertility will not be ameliorated and thereby achieving safer birth and better reproductive health outcomes will almost be near impossible. Therefore it is vital that sexually active women take precaution to promote their sexual health. 


\section{Methods}

\subsection{Search Strategy}

A literature search was conducted on the prevalence of gonorrhea in Nigeria. Relevant citations were identified through a literature search of MEDLINE, PubMed, Google Scholar, and Cochrane Library. The search was based on the combination of the following special index search terms "sexually transmitted infections" OR "sexually transmitted diseases" OR "genital tract infections" OR "reproductive tract infections" OR "gonorrhea" "Neisseria gonorrhoeae" and "Nigeria" "Lists of states in Nigeria" and "prevalence" OR "epidemiology" from 2003 to 2021. Literature search was limited to published studies on humans and duplicated studies were carefully removed. The search was carried out from August 2021- September 2021.

\subsection{Inclusion and Exclusion Criteria}

Studies were selected if (1) they were conducted within Nigeria, (2) study design was cross-sectional and (3) studies reported the prevalence of gonorrhea. A total of six (6) studies were included. Studies were examined for eligibility by reading their titles and abstracts. During the article selection process, studies which did not have full texts were excluded since it was not possible to assess the quality of each article in the absence of their full texts.

\subsection{Characteristics of the Population}

The total population from the various studies analyzed was one thousand seven hundred and twenty five sexually active women between 15 and 45 years. All the women reside in urban areas in Nigeria.

\section{The Way Forward}

In the bid to drastically reduce the spread of gonorrhea, there is significant potential to reduce gonorrhea transmission by scaling up prevention interventions such as the use of contraceptives to prevent the diseases or targeting people with actual cases. Although from the studies reviewed, there were significant reductions in the overall prevalence in Nigeria. However, people with confirmed cases of the infection still engage in risky sexual behaviors with partners with unknown status or negative status. For such cases, it is important to provide a tailored approach that promotes physical, emotional and sexual well- being.

In addition to promoting safer behaviors among people diagnosed with gonorrhea, providing access to and improving the continuity of care is critical. It is clear that $500 \mathrm{mg}$ of intramuscular ceftriaxone is recommended for the treatment of gonorrhea. However, people with confirmed cases do not have access to point of care treatment (Crowell et al., 2017; Gaydos \& Melendez, 2020).Owing that individual clinical outcomes and population-level outcomes can be improved by increasing access to point of care treatment and rates of intramuscular ceftriaxone usage when it is clinically 
indicated, clinicians and relevant bodies should ensure infected people have access to uninterrupted health care that is coordinated and of high quality.

Health education intervention targeted towards women of reproductive age should contain the use of contraceptives or avoidance of indiscriminate sexual intercourse. This would health reduce the incidence of gonorrhea among at risk population and a significant reduction will help reduce the economic and social burden of this infection on individuals, families and larger societies. Additionally a reduction in the incidence of gonorrhea at population level would promote health and well-being.

Studies on the prevalence of gonorrhea in recent times are scare and scanty. More research should be conducted to ascertain the current prevalence of gonorrhea. Further research would give us clearer view of the present situation of gonorrhea in Nigeria and help policymaker evaluate the degree to which behavioral inconsistent adherence to medications or specific interventions may weaken potential gains. The acquired knowledge would help guide policies that bother on gonorrhea prevention and treatment.

In conclusion this review shows that the overall prevalence of gonorrhea among women in Nigeria is declining while the prevalence in some regions has remained stagnated. The paper has presented the reproductive health implication of gonorrhea. Furthermore, it has suggested the way forward in the prevention and treatment of this infection.

\section{References}

Aboyeji, A. P. \& Nwabuisi, C. (2003). Prevalence of sexually transmitted diseases among pregnant women in Ilorin, Nigeria. Journal of Obstetrics and Gynaecology, 23(6), 637-639.

Achalu, I.E. (2004). AIDS and other sexually transmitted diseases: what everyone should know. Smirch Ltd.

Adachi, K., Klausner, J.D., Bristow, C.C., Xu, J., Ank, B., Morgado, M.G., Watts, D.H., Weir, F., Persing, D., Mofenson, L.M. \& Veloso, V.G. (2015). Chlamydia and gonorrhea in HIV-infected pregnant women and infant HIV transmission. Sexually transmitted diseases, 42(10), 554.

Atalabi, O.M., Fayemiwo, S.A., Oladokun, A.A. \& Bakare, R.A., (2013). Pattern of asymptomatic sexually transmitted infections in women undergoing hysterosalpingography for infertility evaluation in Ibadan Nigeria. Tropical Journal of Obstetrics and Gynaecology, 30(2), 91-98.

Barbee, Lindley A., Christine M. Khosropour, Julia C. Dombrowksi, \& Matthew R. Golden. (2017). New HIV diagnosis independently associated with rectal gonorrhea and chlamydia in men who have sex with men. Sexually Transmitted Diseases, 44(7), 385.

Burnett, A. M., Anderson, C. P., \& Zwank, M. D. (2012). Laboratory-confirmed gonorrhea and/or chlamydia rates in clinically diagnosed pelvic inflammatory disease and cervicitis. The American Journal of Emergency Medicine, 30(7), 1114-1117.

Buseri, F. I., Seiyaboh, E. \& Jeremiah, Z. A. (2010). Surveying infections among pregnant women in the Niger Delta, Nigeria. Journal of Global Infectious Diseases, 2(3), 203.

Center for Disease Control (2021). Gonorrhea - CDC Fact Sheet (Detailed Version).Detailed STD Facts - Gonorrhea (cdc.gov).

Chesson, H.W., Kirkcaldy, R.D., Gift, T.L., Owusu-Edusei, K. \& Weinstock, H.S. (2017). An illustration of the potential health and economic benefits of combating antibiotic resistant gonorrhea. Sexually Transmitted Diseases Post Acceptance, 22, 2017. 
Chiwa, M. D., Singh, V. V., Abubakar, M. I. \& Abdalla, E. M. (2019). A statistical survey on awareness and knowledge of sexually transmitted infections [STIs] in north-eastern Nigeria. Journal of statistical and Econometric Methods, 8(2), 27-36.

Cornelisse, V. J., Chow, E. P., Huffam, S., Fairley, C. K., Bissessor, M., De Petra, V. \& Chen, M. Y. (2017). Increased detection of pharyngeal and rectal gonorrhea in men who have sex with men after transition from culture to nucleic acid amplification testing. Sexually Transmitted Diseases, 44(2), 114-117.

Craig, A.P., Gray, R.T., Edwards, J.L., Apicella, M.A., Jennings, M.P., Wilson, D.P. \& Seib, K.L. (2015). The potential impact of vaccination on the prevalence of gonorrhea. Vaccine, 33(36), 4520-4525.

Crowell, T.A., Keshinro, B., Baral, S.D., Schwartz, S.R., Stahlman, S., Nowak, R.G., Adebajo, S., Blattner, W.A., Charurat, M.E., Ake, J.A. \& TRUST/RV368 Study Group (2017). Stigma, access to healthcare, and HIV risks among men who sell sex to men in Nigeria. Journal of the International AIDS Society, 20(1), 21489.

Culbreth, R., Swahn, M. H., Salazar, L. F., Ametewee, L. A. \& Kasirye, R. (2020). Risk factors associated with HIV, sexually transmitted infections (STI), and HIV/STI co-infection among youth living in the slums of Kampala, Uganda. AIDS and Behavior, 24(4), 1023-1031.

Danby, C. S., Cosentino, L. A., Rabe, L. K., Priest, C. L., Damare, K. C., Macio, I. S. \& Hillier, S. L. (2016). Patterns of extragenital chlamydia and gonorrhea in women and men who have sex with men reporting a history of receptive anal intercourse. Sexually Transmitted Diseases, 43(2), 105.

Edem, A., Ntekpe, M. \& Umoekam, N. (2013). Prevalence of syphilis and gonorrhea in patient attending General Hospital, Calabar, Nigeria. International Journal of Modern Biological Medicine, 4(3), 155-168.

Gaydos, C.A. \& Melendez, J.H. (2020). Point-by-point progress: Gonorrhea point of care tests. Expert Review of Molecular Diagnostics, 20(8), 803-813.

Hethcote, H. W. \& Yorke, J. A. (2014). Gonorrhea transmission dynamics and control (Vol. 56). Springer.

Keshinro, B., Crowell, T., Nowak, R., Peel, S., Baral, S., Adebajo, S. \& Ake, J. (2015). High prevalence of HIV, chlamydia, and gonorrhea among men who have sex with men attending trusted community centers in Abuja and Lagos, Nigeria. In Open Forum Infectious Diseases,( 2), 1.

Korenromp, E.L, Wi, L. Resch, S., Stover, J., Broutet, J.(2017). Costing of national STI program implementation for the global STI control strategy for the health sector, 2016-2021PloS One, 12, p. e0170773.

Marrazzo, J. \& Dionne-Odom, J. (2021). Screening for Chlamydia and Gonorrhea. Journal of American Medical Association, 326(10), 913-915.

Mbamara, S. U. \& Obiechina, N. J. (2011). Sero prevalence of venereal disease among pregnant women attending antenatal care (ANC) in Onitsha, Anambra State, Southeast, Nigeria. Nigerian Journal of Medicine: Journal of the National Association of Resident Doctors of Nigeria, 20(1), 57-60.

Mitchell, C. M., Anyalechi, G. E., Cohen, C. R., Haggerty, C. L., Manhart, L. E., \& Hillier, S. L. (2021). Etiology and diagnosis of pelvic inflammatory disease: looking beyond gonorrhea and chlamydia. The Journal of Infectious Diseases, 224(Supplement_2), S29-S35.

Nsofor, C. A. \& Eletuoh, J. (2017). Low prevalence of neisseriagonorrhoeae in Owerri, Nigeria. MOJ Cell Sci Rep, 4(2), 00083.

Okonko, I. O., Okerentugba, P. O., Adejuwon, A. O. \& Onoh, C. C. (2012). Prevalence of sexually transmitted infections (STIs) among attendees of lead city university medical centre in Ibadan, Southwestern Nigeria. Archives of Applied Science Research, 4(2), 980-987.

Omatola, C. A., Iyeh, S. D., Abuh, S. J., Mofolorunsho, C. K., Okolo, M. L. O. \& Akoh, P. Q. (2020). High rate of sexually transmitted infections (STIs) among asymptomatic pregnant women in a resource-poor setting in the middle belt zone of Nigeria. Hosts and Viruses, 7(1), 10-19.

Ramadhani, H. O., Liu, H., Nowak, R. G., Crowell, T. A., Ndomb, T., Gaydos, C. \& Charurat, M. E. (2017). Sexual partner characteristics and incident rectal Neisseria gonorrhea and chlamydia trachomatis infections among gay men and other men who have sex with men (MSM): a prospective cohort in Abuja and Lagos, Nigeria. Sexually Transmitted Infections, 93(5), 348-355. 
Reekie, J., Donovan, B., Guy, R., Hocking, J.S., Kaldor, J.M., Mak, D., Preen, D., Ward, J., Liu, B., Liu, B. \& Preen, D. (2019). Risk of ectopic pregnancy and tubal infertility following gonorrhea and Chlamydia infections. Clinical Infectious Diseases, 69(9), 1621-1623.

Rowley, J., Vander Hoorn, S., Korenromp, E., Low, N., Unemo, M., Abu-Raddad, L, \& Taylor, M. M. (2019). Chlamydia, gonorrhea, trichomoniasis and syphilis: global prevalence and incidence estimates, 2016. Bulletin of the World Health Organization, 97(8), 548.

Tapsall, J. (2005).What is the economic burden imposed by antimicrobial resistance in Neisseria gonorrhea? The growing threat of antibiotic resistance seminar, ReACT group, Uppsala

Umoke, M., Sage, P., Bjoernsen, T., Umoke, P. C. I., Ezeugworie, C., Ejiofor, D. \& Chukwu, O. J. (2021). Co-infection and Risk Factors Associated with STIs among Pregnant Women in Rural Health Facilities in Nigeria: A Retrospective Study. INQUIRY: The Journal of Health Care Organization, Provision, and Financing, (58), 0046958021992912.

Usanga, V. U., Abia-Bassey, L., Inyang-Etoh, P. C., Udoh, S., Ani, F. \& Archibong, E. (2009). Prevalence of sexually transmitted diseases in pregnant and non-pregnant women in Calabar, cross river state, Nigeria. The Internet Journal of Gynecology and Obstetrics, 14(2), 1-7.

Vincent, L.R. \& Jerse, A.E. (2019). Biological feasibility and importance of a gonorrhea vaccine for global public health. Vaccine, 37(50), 7419-7426.

Workowski, K. (2013). Chlamydia and gonorrhea. Annals of Internal Medicine, 158(3), 2-11.

World Health Organization, Global health sector strategy on sexually transmitted infections 2016-2021: Toward Ending STIs, 2016. 